\title{
Investigations on Electronic and Magnetic Properties along with Martensitic Transformation in $\mathrm{Ti}_{2} \mathrm{CoGa}_{1-x} \mathrm{Cu}_{x}$ Alloy
}

\author{
Xiao-Ping Wei*, Xiao-Wei Sun, Ting Song, Jun-Hong Tian and Peng Guo \\ School of Mathematics and Physics, Lanzhou Jiaotong University, Lanzhou 730070, P.R. China
}

(Received December 8, 201\%; in final form April 25, 2018)

\begin{abstract}
Using the coherent potential approximation within full-potential local orbital minimum-basis method, we have performed a systematic investigations on the possible martensitic transformation, electronic and magnetic properties for $\mathrm{Ti}_{2} \mathrm{CoGa}_{1-x} \mathrm{Cu}_{x}$ system. Results show that the system has a potential martensitic transformation and can be synthesized in experiment with exception of $\mathrm{Ti}_{2} \mathrm{CoCu}$ alloy due to their negative formation energy. Calculated total magnetic moment are in good agreement with the Slater-Pauling rule $M_{t}=Z_{t}-18$, thus the system is ferromagnetic half-metallic material except for $\mathrm{Ti}_{2} \mathrm{CoCu}$, which is also confirmed by density of states with the band gap at the Fermi level, a nonmagnetic behavior is observed for $\mathrm{Ti}_{2} \mathrm{CoCu}$ alloy. In addition, the potential martensitic transition from cubic to non-modulated tetragonal transformation illustrates more stability of martensitic phase with respect to cubic one. Such behavior can be explained by the hybridizations between the $d$ orbitals.
\end{abstract}

DOI: 10.12693/APhysPolA.134.519

PACS/topics: 71.15.Mb, 71.20.Lp, 71.20.-b

\section{Introduction}

Recently, the magnetic shape-memory alloys (MSMA) have attracted wide attention due to their unique properties which undergo a variant from the magnetic high temperature austenite phase to tetragonally distorted martensitic phase, and show an extremely large strain in a small external field [1]. The unique property makes these alloys as smart magnetic actuators. So far, the Heusler alloy NiMnGa is found to be the most prominent MSMA due to its large strain of over $5 \%$ in an external field of less than $1 \mathrm{~T}[2-5]$. Althouth there is growing interest in the potential application as a magnetic actuator, commercial realization on NiMnGa has non-negligible shortcomings due to the facts that in the stoichiometric composition it has a martensitic transformation temperature $T_{m}$ of about only $200 \mathrm{~K}$ and a Curie temperature $T_{c}$ of the same order. This encourages the researchers to search for alternative MSMAs with the desired $T_{m}$ and $T_{c}$ above room temperature. Another quantity of interest for MSMA is the saturation magnetization, the large strain can be realized under external magnetic field when there is a substantial saturation magnetization.

$\mathrm{Ti}_{2}$-based Heusler compounds, as a kind of the Heusler materials, have attracted widely attention due to their multiple function such as magnetic shape memory behavior, half-metallicity and thermodynamic properties etc. [6-16]. Among them, some alloys combine the tetragonal distortion with a large magnetic moment and high Curie temperature [17], especially, some of them have synthesized in experiment [18]. In previous work, we

*corresponding author; e-mail: weixp2008@mail.lzjtu.cn demonstrate that $\mathrm{Ti}_{2} \mathrm{CoGa}$ alloy has a potential phase transition from ferromagnetic ordering in austenite to nonmagnetic ordering in martensitic one [17]. However, the drawback is rather large saturation magnetization of the austenite phase.

In current work, the aim is to decrease the saturation magnetization of $\mathrm{Ti}_{2} \mathrm{CoGa}_{1-x} \mathrm{Cu}_{x}$ alloy by $\mathrm{Cu}$ dopant, and then obtaining the system more suitable as MSMA. Furthermore, we will discuss the structural, electronic and magnetic properties along with related martensitic transformation for the system based on first-principles. The paper is organized as follows. Details of computations are given in Sect. 2, followed by the results and detailed discussions in Sect. 3. Finally, in Sect. 4, we summarize our conclusions.

\section{Calculation details}

The calculations are performed by using the fullpotential nonorthogonal local-orbital minimum-basis code (FPLO) $[19,20]$ and employing the coherent potential approximation (CPA) to model $\mathrm{Ti}_{2} \mathrm{CoGa}_{1-x} \mathrm{Cu}_{x}$ system in a random way [21]. The CPA simulation relies on the Blackman-Esterling-Berk theory that includes random off-diagonal matrix elements in the local orbital representation. In our spin resolved, the exchange correlation potential was used in the form of the PerdewBurke-Ernzerhof within the generalized gradient approximation (GGA) [22]. 484 irreducible $k$-points, and the valence orbitals $4 s 4 p 3 d$ for all atoms were used, while the $3 s 3 p$ orbitals are treated as semicore states for transition metal. Left orbitals are as core states. For a self-consistent field iteration, the convergence criterion was set to both the density $\left(10^{-6}\right.$ in code specific units) and the total energy $\left(10^{-8} \mathrm{Ha}\right)$. 


\section{Results and discussion}

\subsection{Structural and energetics related to deformations}

In the present calculations, we calculate the possible martensitic transformation for $\mathrm{Ti}_{2} \mathrm{CoGa}_{1-x} \mathrm{Cu}_{x}$ system with $\mathrm{Hg}_{2} \mathrm{CuTi}$ structure. In the structure, Ti atoms are located at $4 \mathrm{a}$ and $4 \mathrm{c}$ Wyckoff positions, whereas the $4 \mathrm{~b}$ and $4 \mathrm{~d}$ positions are occupied by the $\mathrm{Co}$ and $\mathrm{Ga} / \mathrm{Cu}$ atoms. However, the Ti atoms occupy the $2 \mathrm{a}$ and $2 \mathrm{c}$ positions in martensitic phase, and $\mathrm{Co}$ and $\mathrm{Ga} / \mathrm{Cu}$ are located at $2 \mathrm{~b}$ and $2 \mathrm{~d}$ positions. Initially, we fit the energy-volume relation of ferromagnetic state by using the Murnaghan equation [23] to obtain the equilibrium lattice parameter. Based on the equilibrium states, we further calculate the antiferromagnetic phase since it is reported that introducing dopant may lead to the appearance of antiferromagnetic phase [24]. Calculations show that the antiferromagnetic phase is finally converged to ferromagnetic one when $x=0.00$ and 0.75 , and for other systems the total energies of ferromagnetic state are lower than antiferromagnetic one, thus the system of $\mathrm{Ti}_{2} \mathrm{CoGa}_{1-x} \mathrm{Cu}_{x}$ exhibits a ferromagnetic ground state. Accordingly, the
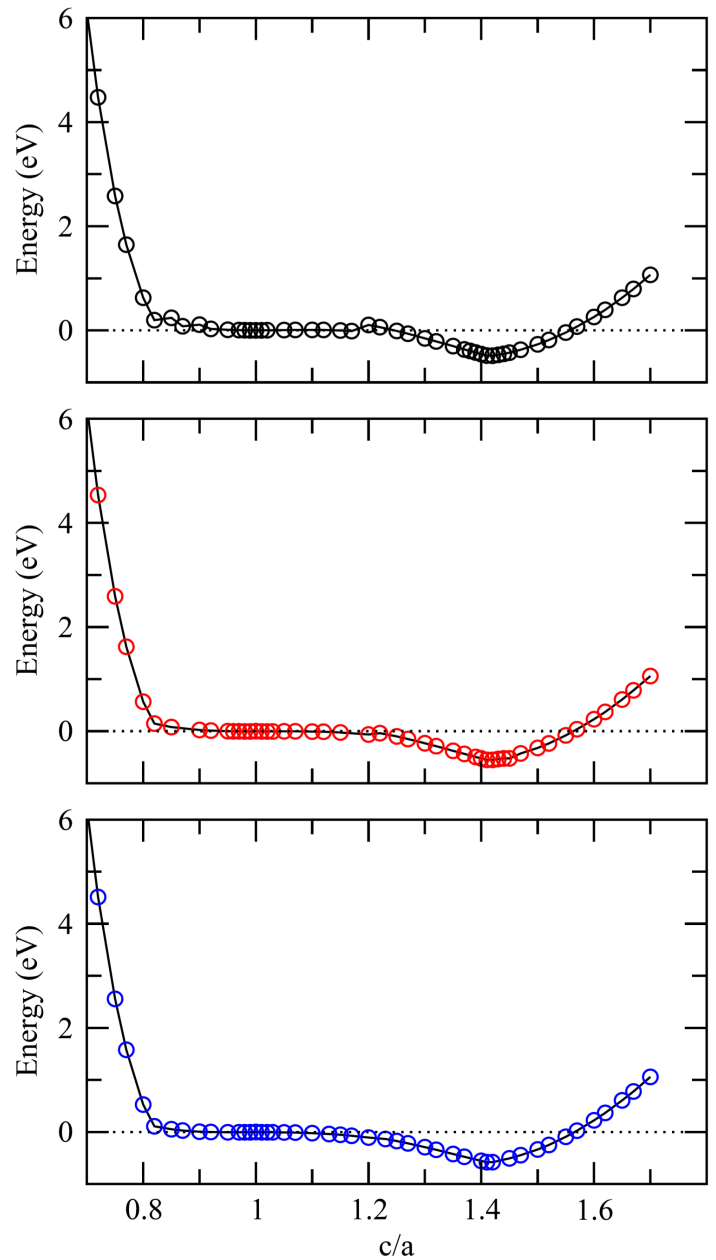

lattice parameters, bulk modulus, the first order derivative of the modulus, total energy are listed in Table I. Herein, we can see the decrease of lattice parameter with the increase of $\mathrm{Cu}$ concentration, which is due to the smaller $\mathrm{Cu}$ atomic radius. The calculated formation energy $E_{f}$ shows that these systems are easier to be formed in the range of 0.75 because of their negative values.

TABLE I

The calculated equilibrium lattice constants $a_{0}$, bulk modulus $B$, the first order derivative of the modulus on volume $B^{\prime}$, formation energy $E_{f}$ and equilibrium total energy $E$ are shown for $\mathrm{Ti}_{2} \mathrm{CoGa}_{1-x} \mathrm{Cu}_{x}$ system with cubic structure.

\begin{tabular}{c|c|c|c|c|c}
\hline \hline $\begin{array}{c}\text { Concentration } \\
x\end{array}$ & $\begin{array}{c}a_{0} \\
{[\AA]}\end{array}$ & $\begin{array}{c}E_{f} \\
{[\mathrm{eV}]}\end{array}$ & $\begin{array}{c}B \\
{[\mathrm{GPa}]}\end{array}$ & $B^{\prime}$ & $\begin{array}{c}E \\
{[\mathrm{Ha}]}\end{array}$ \\
\hline 0.00 & 6.197 & -0.827 & 64.3 & 2.23 & -5045.238 \\
0.25 & 6.125 & -0.489 & 62.6 & 4.45 & -4972.956 \\
0.50 & 6.091 & -0.204 & 72.0 & 2.93 & -4900.676 \\
0.75 & 6.040 & -0.029 & 69.4 & 4.51 & -4828.400 \\
1.00 & 6.002 & 0.064 & 70.3 & 4.39 & -4756.127
\end{tabular}
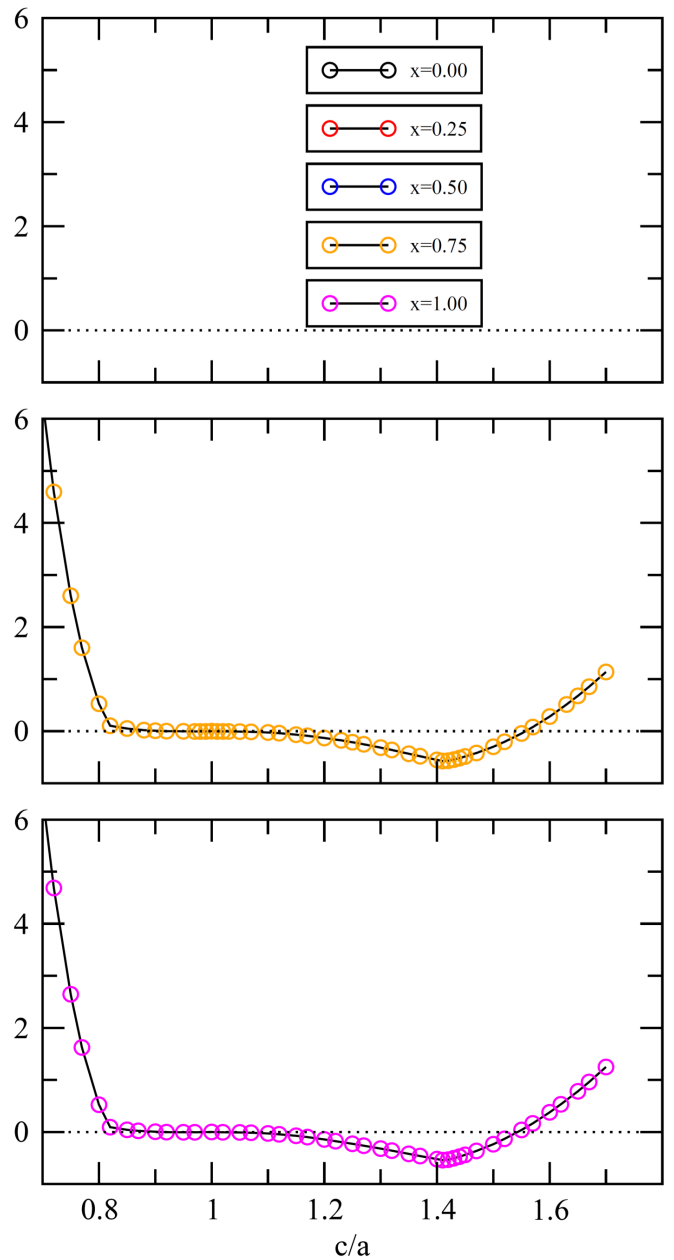

Fig. 1. Total energy per formula unit as a function of tetragonal deformations $(c / a)$ for the $\mathrm{Ti}_{2} \mathrm{CoGa}_{1-x} \mathrm{Cu}_{x}$ system. The energies are plotted with reference to the energy of the cubic phase. 
TABLE II

The calculated total $M_{\text {tot }}$ and partial magnetic moments of different Wyckoff sites for $\mathrm{Ti}_{2} \mathrm{CoGa}_{1-x} \mathrm{Cu}_{x}$ system.

\begin{tabular}{c|c|c|c|c|c|c|c|c|c|c}
\hline \hline Concentration & \multicolumn{9}{|c|}{ Cubic } & \multicolumn{5}{c}{ Tetragonal } \\
\hline \multirow{2}{*}{$x$} & $\begin{array}{c}M_{\text {tot }} \\
{\left[\mu_{B}\right]}\end{array}$ & $\begin{array}{c}\mathrm{Ti}_{4 a} \\
{\left[\mu_{B}\right]}\end{array}$ & $\begin{array}{c}\mathrm{Ti}_{4 c} \\
{\left[\mu_{B}\right]}\end{array}$ & $\begin{array}{c}\mathrm{Co}_{4 b} \\
{\left[\mu_{B}\right]}\end{array}$ & $\begin{array}{c}(\mathrm{Ga} / \mathrm{Cu})_{4 d} \\
{\left[\mu_{B}\right]}\end{array}$ & $\begin{array}{c}M_{\text {tot }} \\
{\left[\mu_{B}\right]}\end{array}$ & $\begin{array}{c}\mathrm{Ti}_{2 a} \\
{\left[\mu_{B}\right]}\end{array}$ & $\begin{array}{c}\mathrm{Ti}_{2 c} \\
{\left[\mu_{B}\right]}\end{array}$ & $\begin{array}{c}\mathrm{Co}_{2 b} \\
{\left[\mu_{B}\right]}\end{array}$ & $\begin{array}{c}(\mathrm{Ga} / \mathrm{Cu})_{2 d} \\
{\left[\mu_{B}\right]}\end{array}$ \\
\hline 0.00 & 2.000 & 1.378 & 0.860 & -0.203 & -0.035 & 0.000 & 0.000 & 0.000 & 0.000 & 0.000 \\
0.25 & 1.500 & 1.120 & 0.752 & -0.335 & $-0.034 /-0.049$ & 0.000 & 0.000 & 0.000 & 0.000 & 0.000 \\
0.50 & 1.000 & 0.804 & 0.569 & -0.342 & $-0.027 /-0.036$ & 0.000 & 0.000 & 0.000 & 0.000 & 0.000 \\
0.75 & 0.499 & 0.427 & 0.319 & -0.231 & $-0.015 /-0.017$ & 0.000 & 0.000 & 0.000 & 0.000 & 0.000 \\
1.00 & 0.000 & 0.000 & 0.000 & -0.000 & 0.000 & 0.000 & 0.000 & 0.000 & 0.000 & 0.000
\end{tabular}

In order to study the tetragonal distortions of $\mathrm{Ti}_{2} \mathrm{CoGa}_{1-x} \mathrm{Cu}_{x}$ system. In Fig. 1 , we present the function of total energy as a $c / a$ ratio, the aim is to see whether a tetragonal non-modulated phase is energetically lower than the cubic one, the possible phase transition happens only when total energy of tetragonal phase is lower than one of cubic phase. To this end, total energy calculations at different $c / a$ ratio are performed as an estimation of the tetragonal transformation. By minimizing the total energy with respect to $c / a$, we find the lowest energy of tetragonal phase, implying a potential transition of martensitic phase. The energy minimum of tetragonal phase, when $x$ is 0.00 and 0.25 , is pinned at 1.42 , the rest remains at 1.41 .

\subsection{Magnetic moments}

The calculated total and partial magnetic moments are given in Table II. For $\mathrm{Ti}_{2} \mathrm{CoGa}_{1-x} \mathrm{Cu}_{x}$ system, Ti atoms couple ferromagnetically to each other. In contrast, the magnetic moments of $\mathrm{Ti}$ atoms couple antiferromagnetically with Co atom. In addition, the magnetic moments are mainly carried by $\mathrm{Ti}$ atoms, thus resulting in a ferromagnetic ground state in cubic phase. Moreover, we plot the function of total magnetic moment on the valence electron number or $\mathrm{Cu}$ concentration in Fig. 2, it finds that the calculated magnetic moments are in good agreement with the Slater-Pauling rule $M_{t}=Z_{t}-18$ [25], where $M_{t}$ is total spin magnetic moment, and $Z_{t}$ is valence electron number in unit cell. In the martensitic phase of different $\mathrm{Cu}$ concentration, the magnetic moment disappears, suggesting a nonmagnetic nature. In order to explain the trends in energy, we will analyze the differences in the electronic density of states (DOS) for the system. Such an analysis will be presented in the next subsection.

\subsection{Density of states}

In what follows, we present the spin-polarized total DOS for $\mathrm{Ti}_{2} \mathrm{CoGa}_{1-x} \mathrm{Cu}_{x}$ system in Fig. 3, which contains the DOS in cubic and martensitic phases at different $\mathrm{Cu}$ concentration. For both spin configuration of cubic phase, we can observe that the majority-spin states show the semiconductor character when $x \leq 0.75$, and the minority-spin states preserve the metallic nature, thus these systems indicate half-metallic properties, that is also confirmed by their total spin magnetic moment.
The system reveals nonmagnetic character owing to the same weight of majority-spin and minority-spin states when the $\mathrm{Ga}$ atom is replaced fully by $\mathrm{Cu}$ atom, it is also confirmed by calculated total magnetic moment, i.e., the total spin magnetic moment is closed to zero. In addition, we also notice the band gap of minority-spin states become smaller and smaller as the increase of $\mathrm{Cu}$ concentration because of reduction of exchange splitting energy. In majority-spin states, the Fermi level slowly move from the pseudogap to the shoulder states, also illustrating the increase of formation energy. In fact, the contribution to overall DOS is mainly from transition metal $d$ orbitals and $p$ orbital of Ga atom.

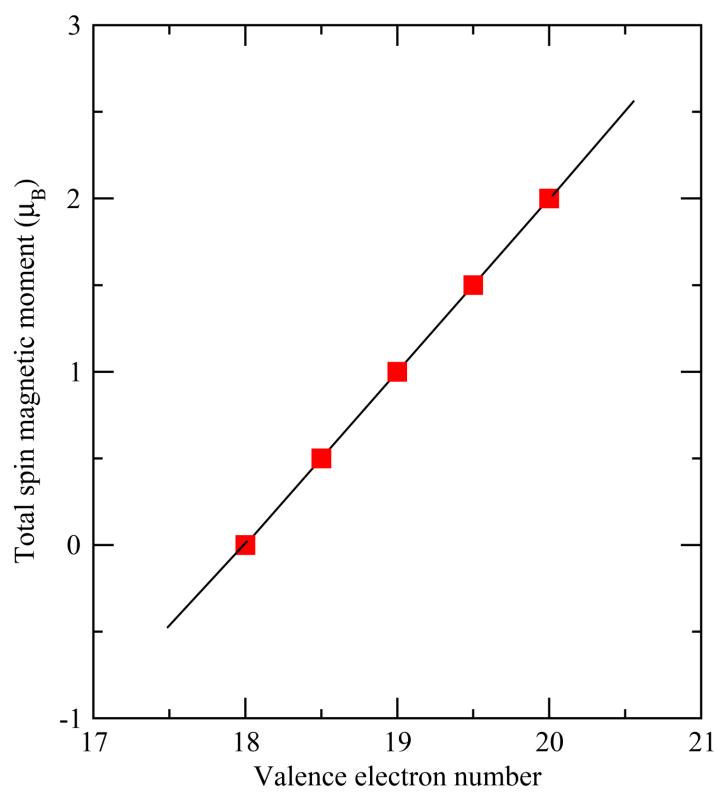

Fig. 2. Slater-Pauling rule between total spin magnetic moment and valence electron number for $\mathrm{Ti}_{2} \mathrm{CoGa}_{1-x} \mathrm{Cu}_{x}$ system.

A comparison of the total DOS of cubic phase with respect to martensitic phase is made in Fig. 3, we can see that the electronic states of their martensitic phase present the nonmagnetic properties due to the same weight of majority-spin and minorityspin states. However, some marginal differences are still obvious around the Fermi level. Most noticeably, the peaks of DOS are more prominent for martensitic phase of $\mathrm{Ti}_{2} \mathrm{CoGa}$, indicating 


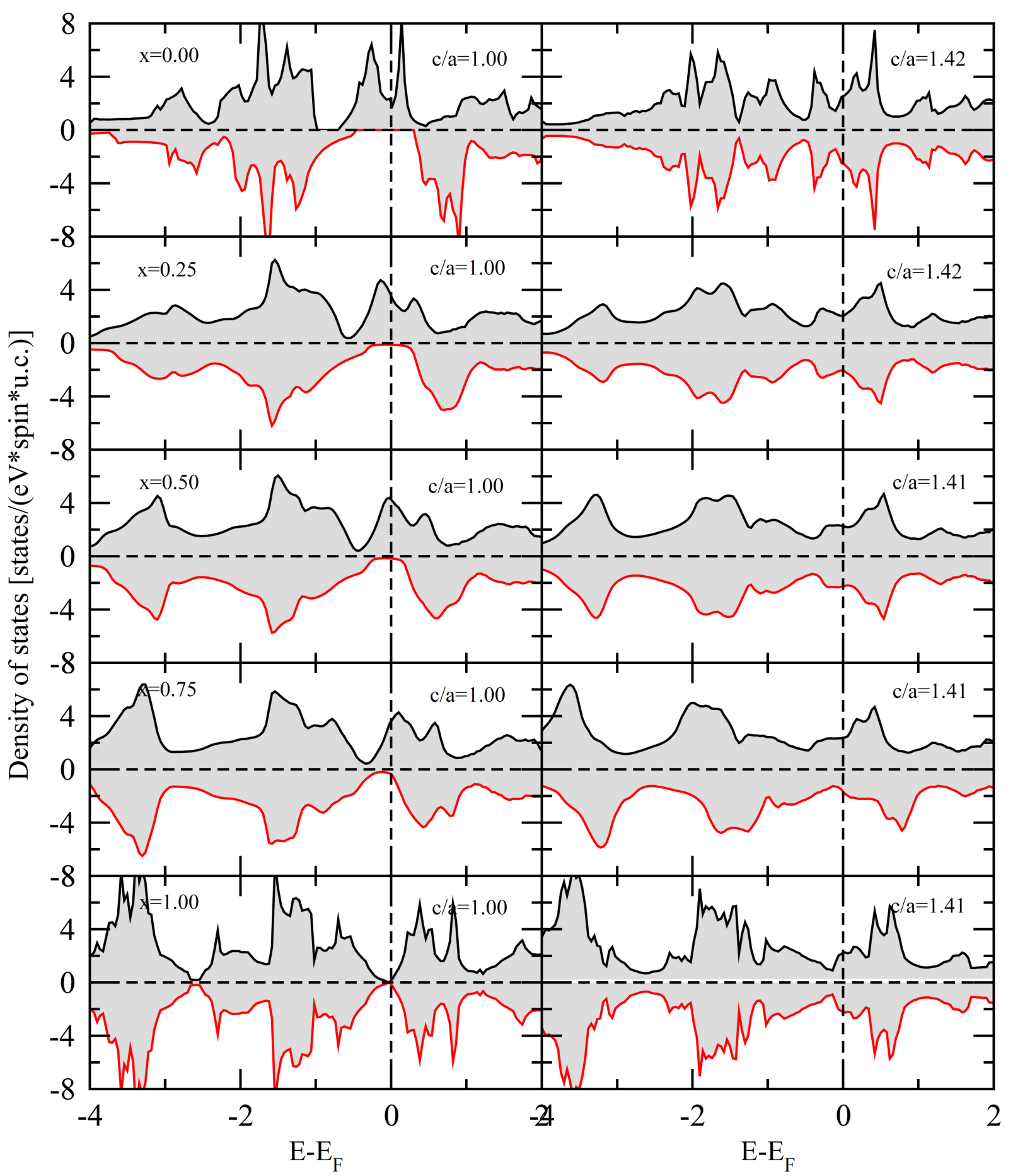

Fig. 3. Spin-polarized total densities of states of $\mathrm{Ti}_{2} \mathrm{CoGa}_{1-x} \mathrm{Cu}_{x}$ system calculated at their cubic phase and at the global stable martensitic phase. Note that the Fermi energy is defined as the zero energy.

the stronger localization of $d$ electrons. Another noteworthy feature of the total DOS in the tetragonal structure has few changes. As an increase of $\mathrm{Cu}$ concentration, resulting in transforming the located spin peaks into multiple peaks with reduced intensities and an overall shift of states toward lower energies. Due to such migrations of states, the valleys in the both spin states get filled, and then produce gentle peaks in the DOS. These migrations of electronic states toward lower energies stabilize the tetragonal phase compared to their cubic phase. In- 
spection of the DOS for the stable tetragonal structure indicate better stabilities to the cubic phases due to migrations of states toward lower energies. This explains why the tetragonal phases are stable energetically and marginally lower than the cubic phases.

\section{Summary and conclusions}

We have performed systematical investigations on possible martensitic phase, electronic and magnetic properties of $\mathrm{Ti}_{2} \mathrm{CoGa}_{1-x} \mathrm{Cu}_{x}$ system by using FPLO-CPA method. For cubic structure, calculations show that the $\mathrm{Ti}_{2} \mathrm{CoGa}_{1-x} \mathrm{Cu}_{x}$ system can be synthesized in experiment with exception of $\mathrm{Ti}_{2} \mathrm{CoCu}$ alloy due to their negative values of formation energy. As the increase of $\mathrm{Cu}$ concentration, the lattice parameter of system decreases because of smaller atomic radius for $\mathrm{Cu}$ atom, and the total energy is increasing owing to lower electronegativity of $\mathrm{Cu}$ atom. Calculated total magnetic moments are in good agreement with the Slater-Pauling rule $M_{t}=Z_{t}-18$, which is firmed by the total DOS, i.e., one of spin channels indicates the half-metallic character. Especially, the nonmagnetic behavior is observed for $\mathrm{Ti}_{2} \mathrm{CoCu}$ alloy. In addition, we find that the system exhibit potential martensitic transition from cubic to non-modulated tetragonal transformation according to the function of total energy on deformation, suggesting more stability of martensitic phase with respect to cubic one. Such behavior can be explained by the hybridizations between the $d$ orbitals. For martensitic phase at different $\mathrm{Cu}$ concentration, the stable structures are located around $c / a=1.41$, meanwhile they indicate the nonmagnetic behavior due to the same weight of DOS in both configuration.

\section{Acknowledgments}

The Project is supported by the Young Scholars Science Foundation of Lanzhou Jiaotong University (No. 2015028). This work is also supported by National Natural Science Foundation of China (No. 11647151) and the Scientific Research Project of Gansu Province (No. 2016-B029).

\section{References}

[1] P.J. Brown, A.Y. Bargawi, J. Crangle, K.-U. Neumann, K.R.A. Ziebeck, J. Phys. Condens. Matter 11, 4715 (1999).

[2] K. Ullakko, J.K. Huang, C. Kanter, V.V. Kokorin, R.C. O'Handley, Appl. Phys. Lett. 69, 1966 (1996).
[3] V.A. Chernenko, V.V. Kokorin, O.M. Babii, I.K. Zasimchuk, Intermetallics 6, 29 (1998).

[4] S.J. Murray, M. Marioni, S.M. Allen, R.C. O'Handley, T.A. Lograsso, Appl. Phys. Lett. 77, 886 (2000).

[5] X. Jin, M. Marioni, D. Bono, S.M. Allen, R.C. O'Handley, T.Y. Hsu, J. Appl. Phys. 91, 8222 (2002).

[6] X.P. Wei, J.B. Deng, G.Y. Mao, S.B. Chu, X.R. Hu,. Intermetallics 29, 86 (2012).

[7] L. Zhang, L.Y. Wang, J.J. Lu, X.T. Wang, L. Wang, J. Kor. Phys. Soc. 65, 2058 (2014).

[8] F. Ahmadian, J. Kor. Phys. Soc. 64, 277 (2014).

[9] Moaid K. Hussain, G.Y. Gao, Kai-Lun Yao, Int. J. Mod. Phys. B 29, 1550175 (2015).

[10] S. Kervan, N. Kervan, J. Electron. Mater. 41, 1978 (2012).

[11] X.P. Wei, Y.D. Chu, X.W. Sun, J.B. Deng, Eur. Phys. J. B 86, 450 (2013).

[12] Moaid K. Hussain, G.Y. Gao, Kai-Lun Yao, J. Supercond. Nov. Magn. 28, 3285 (2015).

[13] B. Hamri, Abdelkrim Hamri, Aida Hamri, A. Hallouche, B. Abbar, Int. J. Mod. Phys. B 30, 1650076 (2016).

[14] Y. Feng, B. Wu, H.K. Yuan, A.L. Kuang, H. Chen, J. Alloys Compd. 557, 202 (2013).

[15] P. Lukashev, P. Kharel, S. Gilbert, B. Staten, N. Hurley, R. Fuglsby, Y. Huh, S. Valloppilly, W. Zhang, K. Yang, R. Skomski, D.J. Sellmyer, Appl. Phys. Lett. 108, 141901 (2016).

[16] Q.L. Fang, J.M. Zhang, K.W. Xu, J. Magn. Magn. Mater. 349, 104 (2014).

[17] X.P. Wei, Y.D. Chu, X.W. Sun, Y. E, J.B. Deng, Y.Z. Xing, Mater. Res. Bull. 62, 212 (2015).

[18] W.W. Feng, X. Fu, C.H. Wan, Z.H. Yuan, X.F. Han, Nguyen Van Quang, Sunglae Cho, Phys. Status Solidi $R R L$ 9, 641 (2015).

[19] K. Koepernik, H. Eschrig, Phys. Rev. B 59, 1743 (1999).

[20] I. Opahle, K. Koepernik, H. Eschrig, Phys. Rev. B 60, 14035 (1999).

[21] K. Koepernik, B. Velicky, R. Hayn, H. Eschrig, Phys. Rev. B 55, 5717 (1997).

[22] J.P. Perdew, Y. Wang, Phys. Rev. B 45, 13244 (1992).

[23] F.D. Murnaghan, Finite Deformation of an Elastic Solid, Dover, New York 1967.

[24] J.E. Douglas, J.C. Castillo, P. Adler, E.E. Levin, S. Krämer, K.L. Page, C. Felser, T.M. Pollock, R. Seshadri, Phys. Rev. B 94, 094412 (2016).

[25] S. Skaftouros, K. Özdoğan, E. Şaşığlu, I. Galanakis, Phys. Rev. B 87, 024420 (2013). 